\title{
Multi-point Shape optimization of a horizontal axis tidal stream turbine
}

\author{
Hassan el sheshtawy, Ould el Moctar, Satish Natarajan \\ University of Duisburg-Essen, Institute of Ship Technology \\ Ocean Engineering and Transport Systems, Bismarckstrasse 69, 47057 Duisburg, Germany
}

\begin{abstract}
A method was developed to perform shape optimization of a tidal stream turbine hydrofoil using a multi-objective genetic algorithm. A bezier curve parameterized the refrence hydrofoil profoil NACA 63815. Shape optimization of this hydrofoil maximized its lift-to-darg ratio and minimized its pressure coefficient, thereby increasing the turbines power output power and improving its cavitation characteristics. The Elitist Non-dominated Sorting Genetic Algorithm (NSGA-II) was employed to perform the shape optimization. A comparative study of two-and three-dimensional optimizations was carried out. The effect of varing the angle of attack on the quality of optimized results was also studied. predictions based on two-dimensional panel method results was also studied. Preditions based on a two-dimensional panel method and on a computational fluid dynamics code were compared to experimental measurments.
\end{abstract}

\section{Keywords}

Hydrofoil optimization, NSGA-II, CFD, XFOIL, NACA 63815, Bezier curve

\section{Introduction}

The demand for renewable energy has been increasing over the previous decade. Renewable energy resources are available in various forms; one of them is tidal energy. Tidal energy can be harnessed in a number of ways, one being the use of tidal stream turbines. Therefore, was optimized the shape of the subject hydrofoil itself to improve the performance. The optimization proicess can be throught of as making the best use of resources under giving constraints. Many 
researchers performed a two-dimensional shape optimization of airfoils for aircraft (Shahrokhi and Jahangirian, 2007; Peigin and Epstein, 2004; Nemec et al., 2004) and others for wind turbines (Ribeiro et al., 2012; Mukesh et al., 2014). For wind turbines, airfoils can be operated at a high angle of attack (AOA), even undar stall conditions, which is not the case for aircraft. The lift-to-darg ratio (L/D) or glide ratio (GR) are the most important criteria for shape optimization of wind turbine airfoil (Ribeiro et al., 2012). The same methods and alogrithms that optimize airfoils can be used top optimize hydrofoils. However, the parameters to be optimized differ because every turbomachinary has its own optimization targets that should be taken into accouint. Besides the L/D ratio, caviation characteristics should be considered to perform shape optimization of hydrofoil((Luo et al., 2014)). Liu and Veitch (2012) develpoed a code to predict the strength of wind turbine blades and to evaluate the maximum balde thickness. They validated their code against model and full-scale experimental measurments. Besides increasing the strength of the turbine blade. Goundar and Ahmed (2013) designed hydrofoils for different sections of an HATST to maximize its L/D ratio and to reduce the possibility of the occurance of caviation.

Paolo et al. (2016) optimized marine propeller hydrofoils by taking into account the effect of cavitation. They parameterized the hydrofoil using B-Splines (De Boor et al., 1978), and carried out shape optimization using NSGA-II (Deb et al., 2002a) to widen the cavitation bucket of parent hydrofoil and to maximize L/D ratio. To this end, they employed two different flow solver approaches, namely, viscid and inviscid. The author investigated the influence of optimization algorithms such as NSGA-II and Particle Swarm Optimization (PSO) (Kennedy and Eberhart, 1995; Kennedy, 2011) on the performance of marine propeller design. Three modified optimization algorithms were proposed, two of which were based on NSGA-II algorithm that was modified by a meta-model and one of which is non-dominated PSO algorithm that is a combination of NSGA-II algorithm and standard PSO algorithm. Tests were carried out on a real-life propeller for naval application. The propeller geometry was described by Rolls-Royce distribution curves which can be controlled by input parameters such as blade area ratio, skew or rake at the blade tip. Further, B-splines were used to change the geometry of the blade locally. Among the two algorithms presented that utilize a meta-model, the performance of SANA NSGA-II was comparably better and the fastest and strictest convergence towards the Pareto front was observed in Non-dominated sorting PSO algorithm. The most important criterion for the marine propellers is to reduce the possibility of cavitation occurrence and to maximize the L/D ratio. Some researchers performed shape optimization of hydrofoils for marine applications. ChingYeh et al. (2006); Ouyang et al. (2006) performed shape optimisation for increasing the GR. Apart from GR, Zhang et al. (2016) also evaluated cavitation characteristics of the optimised hydrofoil using Computational Fluid Dynamics (CFD). Litvinov (1995) optimized the shape of a hydrofoil, which moves with low velocity in a viscous incompressible fluid, to minimize the power needed to move it by imposing constraints on lift force, area, and geometry of the hydrofoil. Goundar et al. (2012) studied the hydrodynamic characteristics of HF-Sx hydrofoil experimentally and numerically, and compared their performance to other hydrofoils of marine current turbines. Furthermore, they designed a hydrofoil to work well at TSRs from 3.0 to 4.0 
without decreasing the possibility of cavitation. Tahani and Babayan (2017) carried out shape optimization for horizontal axis turbines to increase its power coefficient by employing Ant Colony Optimization (ACO) algorithm, and they calculated the power coefficient using Blade Element Momentum Theory (BEMT). Luo et al. (2014) optimised the shape of a hydrofoil for marine current turbines to increase its L/D ratio and to improve its cavitation performance over a wide range of AOA. They parameterised the hydrofoil using Bezier curve and carried out optimisation by employing NSGA-II. Furthermore, they developed a FORTRAN code and coupled it with CFD solver. The optimization method proposed in the paper effectively improves the L/D performance of the hydrofoil from AOA $0^{\circ}$ to $12^{\circ}$. The cavitation performance was mainly improved from $\mathrm{AOA} 6^{\circ}$ to $12^{\circ}$ with little improvement from $0^{\circ}$ to $6^{\circ}$.

In the present paper, we carried out shape optimisation of a tidal stream turbine hydrofoil to improve its output power. We developed a Matlab code to optimize the shape of its hydrofoil and coupled our code with a flow solver to determine the turbine's performance. We chose NSGAII as the optimisation algorithm and carried out a comparative two- and three- dimensional optimization study. Furthermore, we investigated the effect of angle of attack on the quality of optimisation results.

\section{Optimization Methodology}

Optimization algorithms can be categorized into two groups, namely, genetic based methods and heuristic alorithms. Genetic based methods usually require derivatives of the objective functions to guide the search process. Although efficient, these methods do not always converge to global optima (Ribeiro et al., 2012). The convergence of the genetic based methods depends on the initial guess. Heuristic algorithms, on the other hand, are slower, but they converge to global optima regardless of the initial guess. Objective functions alone suffice for the optimization. A Genetic Algorithm (GA) is the most popular of the heuristic algorithms. The GAs fall into two categories, namely, single- and multi- objective GAs. For practical engineering applications, it is unrealistic to perform optimizations based on only one objective and to ignore the others.

It is prudent to consider all relevant parameters (Konak et al., 2006). The GAs are optimization techniques that solve nonlinear or non-differentiable optimization problems. They start with an initial set of randomly generated solutions, which are then compared with the objective function. Subsequent gernerations are generated from previous generations. We developed a new code for two- and three- dimensional shape optimizations based on the Elitist Non- dominated Sorting Genatic Algorithm (NSGA-II), using the code of (Baskar et al., 2015) as the starting point. We incorporated another set of codes that parameterized the reference hydrofoil to constrain the shape of the generated hydrofoils. This limited the upper and lower bounds of the generated hydrofoils and evaluated the crowding distance for the three-dimensional optimization. Then we modified the conditions for a non-dominating sorting of the optimized hydrofoils. Finally, we coupled this code with the flow solver and added a post processing liberary to rearrange the results to meet our requirments. Figure 1 schematically depicts our optimization 


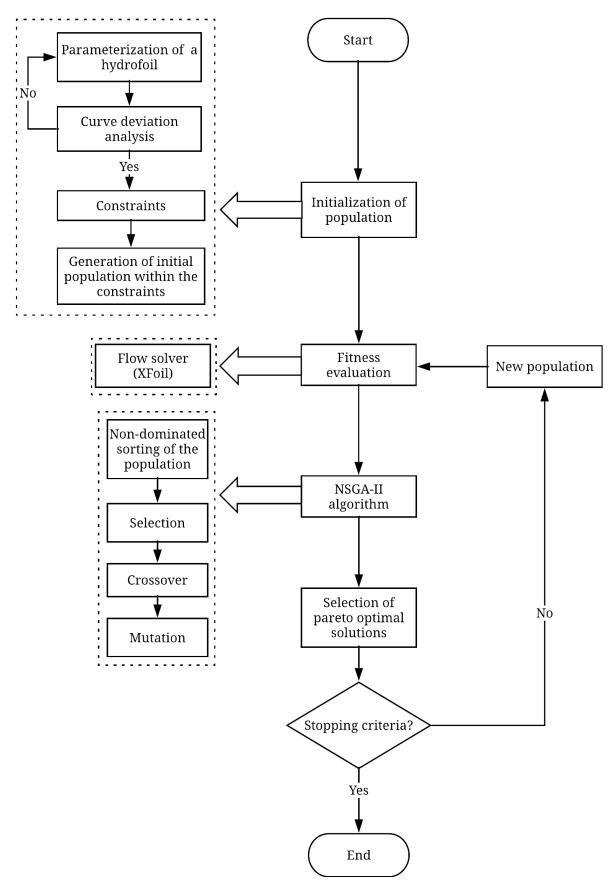

Figure 1: Flowchart of optimization methodology

method.

\subsection{Initialization of population}

The initialization of population was done in three steps: parameterization of the reference hydrofoil, definition of constraints and generation of initial population. Here, parameterization refers to the process of representing the hydrofoil shape mathematically and determining its control points, which in turn can vary and control the shape of the hydrofoils. In this study, the reference hydrofoil was parameterized using Bezier curve. A Bezier curve of order $n$ is defined by:

$$
P(t)=\sum_{i=0}^{n} B_{i, n} Q_{i}=\sum_{i=0}^{n} C_{n}^{i}(1-t)^{n-i} t^{i} Q_{i}
$$

where $P(t)$ is a point on the Bezier curve, $t \in[0,1]$ is the weight coefficient of the point on the Bezier curve, $B_{i, n}$ is the Bernstein basis function, $Q_{i}$ is the $i^{\text {th }}$ control vertex of the Bezier curve, and $C_{n}^{i}$ is the combinatorial symbol (Luo et al., 2014). The hydrofoil NACA 63815 (Abbott and Von Doenhoff, 1959) was selected as the reference hydrofoil since it was assessed to be cavitation free through experiments (Batten et al., 2006). Figure 2 the original and parametrized 


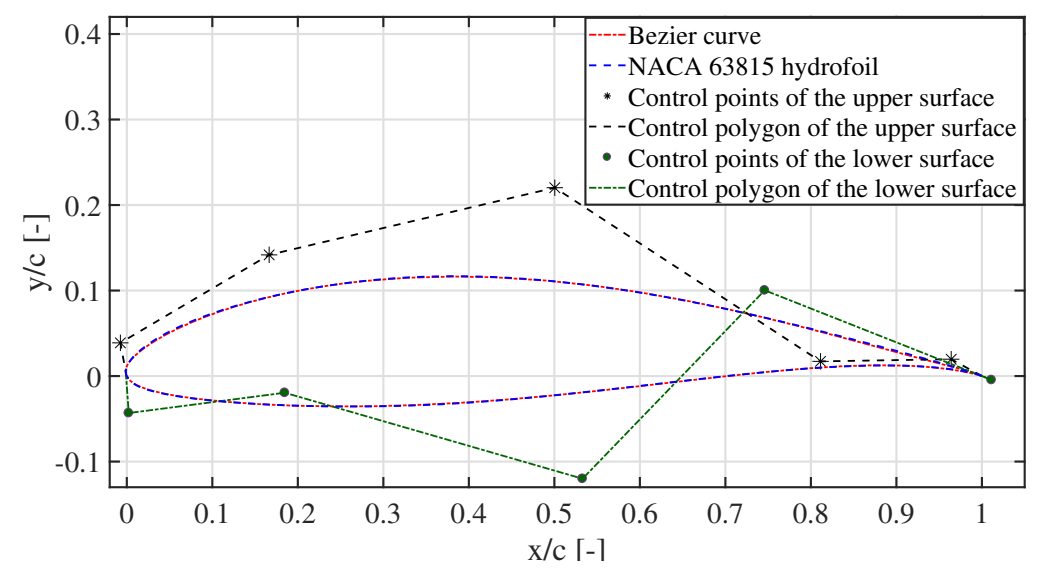

Figure 2: Parameterization of NACA 63815 hydrofoil using Bezier curve, where X/C and Y/C are the normalized distance in $\mathrm{x}$ and $\mathrm{y}$ directions to the hydrofoil chord length ratio

hydrofoil are shown. It can be seen that the Bezier curve represents the hydrofoil accurately and by moving the control points, the shape of the hydrofoil can be varied and controlled. In order to determine mathematically how effectively the reference hydrofoil was parameterized, curve deviation analysis (Luo et al., 2014) was performed using the formula below:

$$
\delta=\frac{\Delta_{i}}{l}
$$

where $\delta$ is the normalized deviation of the Y-coordinates of the Bezier curve from that of the reference hydrofoil at any point $i, \Delta_{i}$ is the deviation value of the parameterized hydrofoil with respect to the reference hydrofoil at $\mathrm{i}, l$ is the chord length of the original hydrofoil. It can be seen in Figure 3 that $\delta$ of the upper and the lower surfaces over X/C are less than $1 \times 10^{-3}$ and $2.2 \times 10^{-3}$ respectively. The deviation is negligible and hence, the parameterization technique is accurate enough to represent the reference hydrofoil and to carry out shape optimization. To maintain a realistic shape of the hydrofoil, constraints on its thickness are employed as shown in Figure 4. A major concern with using flow solvers is to make the hydrofoil shape smooth enough, which helps to get fast converge solution (Prosser et al., 2010). Another concern is that if the upper and the lower bounds are too wide or too narrow, the hydrofoil may not be continuous at the leading edge. Therefore, the constraints must be chosen with care. The maximum thicknesses of both the pressure and suction sides are allowed to increase and decrease by $40 \%$ and $30 \%$ respectively with respect to its chord. Here, the upper and lower bounds were chosen through trial and error on flow solver. The constraints have been particularly limited near the trailing edge to ensure that the curves of the pressure and suction sides do not intersect during optimization. The leading and trailing edge coordinates of hydrofoil are fixed. Further, the $\mathrm{x}$-axis coordinates of all the control points are fixed. Only the $y$-coordinates of the ten control points shown in Figure 2 are allowed to vary during optimization. 


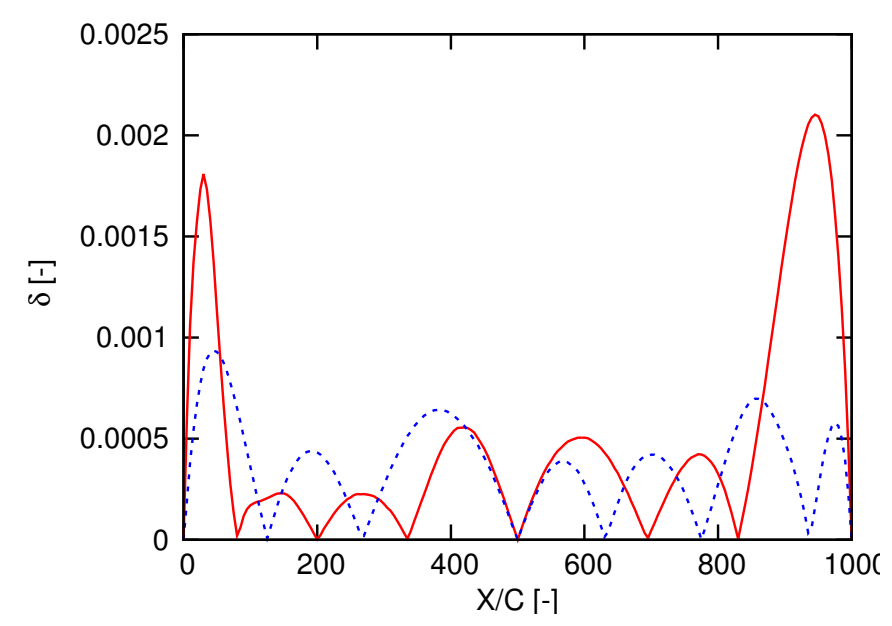

Figure 3: Curve deviation analysis of the parameterized hydrofoil, where the dotted blue line represents the deviation in the suction side and the solid red line represents the deviation in the pressure side.

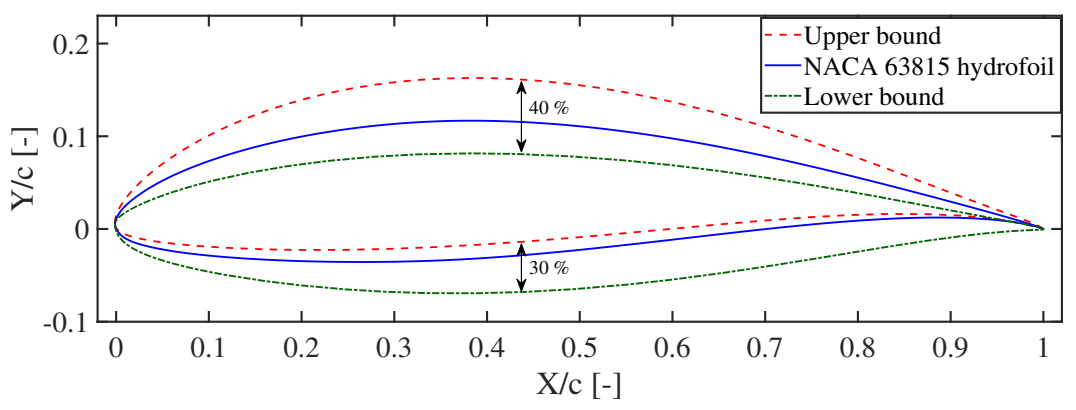

Figure 4: Optimized hydrofoil with upper and lower bounds for the optimization 
(a)

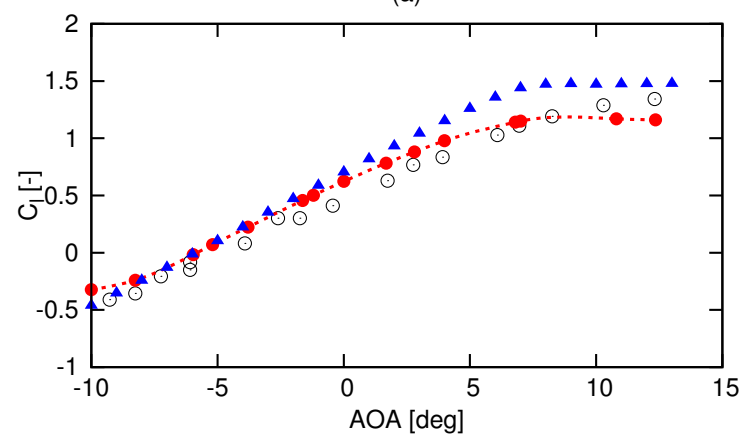

(b)

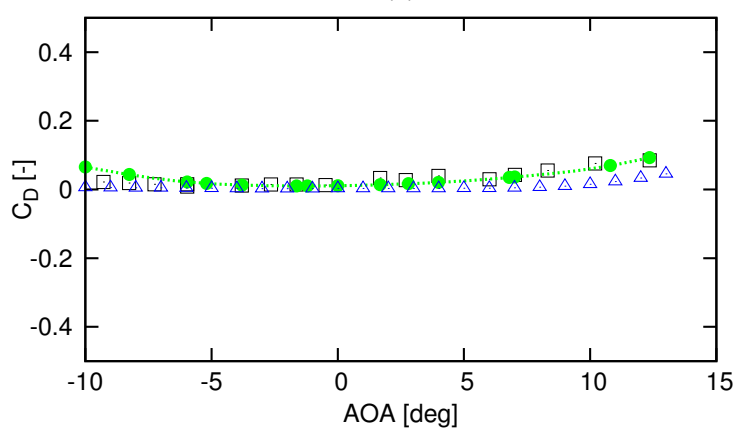

Figure 5: Comparision of CFD and XFOIL results with the experimental measurments, where (a) Lift coefficients $\left(C_{l}\right)$ and (b) drag coefficients $\left(C_{d}\right)$ at different AOA

\subsection{The flow solver}

Some researchers use Computational Fluid Dynamics and a lot of others use 2-D panel code, for instance, XFOIL (Drela, 1989) as the flow solver. In this study we have compared the results of both the methods with the experimental data. The experimental results of performance characteristics of NACA 63815 were obtained from (Molland et al., 2004) for a Reynolds number of $0.8 \times 10^{6}$ and for a flow velocity of $3.492 \mathrm{~m} / \mathrm{s}$. The computational domain for evaluating the performance characteristics of the hydrofoil using CFD simulation was set as described in (Luo et al., 2014). The CFD simulation was performed in a $2 \mathrm{D}$ environment. The mesh size was set to 198000 cells with a $\mathrm{y}^{+}$value of 0.88 . The $k-\omega$ SST turbulence model (Menter, 1993) was chosen to close RANS equations. The SIMPLE algorithm (Patankar, 2016) was used for pressure-velocity coupling. Fifteen different Angles Of Attack (AOA) ranging from $-10^{\circ}$ to $12.5^{\circ}$ were considered for the CFD simulations. The Reynolds number in XFOIL was set to $8 \times 10^{5}$ and the maximum number of iterations for the analysis of each of the hydrofoils was set to 500. The performance characteristics were evaluated in viscous mode. As shown in Figure 5, the CFD results are closer to the experimental measurements than that of XFOIL. However, the computational time using CFD was approximately 4 hours, while using XFOIL it were merely 90 seconds. We tested almost 2480 optimized hydrofoils (30 generations and each generation has 80 hydrofoils) and to this end we needed a method that gives acceptable results within a short span of time. Furthermore, the performance predictions of XFOIL were also found to match the experimental results closely and it is also simple to use. Therefore, XFOIL was chosen as the flow solver (Zhang et al., 2016; Batten et al., 2006, 2007; Goundar et al., 2012; Goundar and Ahmed, 2013).

\subsection{Optimization algorithm}

In this algorithm the solutions are sequenced based on their degree of non-domination and sent to the next generation. A simple GA consists of three genetic operators: selection, crossover 
and mutation. Selection is a process in which pairs of candidate solutions are selected to reproduce. The pairs are selected based on their fitness scores. This operator is an artificial version of Darwinian's natural selection. Tournament selection method has been used to perform the selection operation. This method was chosen since it has better convergence and computational time compared to a lot of other methods. In this method, two candidate solutions are picked randomly and their ranks are compared. The candidate solution that has better rank is selected and the other one is rejected. This process continues until all the candidate solutions are analyzed. Here, two hydrofoils are picked randomly and their lift, drag and minimum pressure coefficients are analysed. In GA, the next generation solutions are created by exchanging information among strings of the previous generation. This process is known as crossover and it is equivalent to reproduction in evolutionary biology. The crossover operation was performed by Simulated Binary Crossover(SBX). Mutation is the process in which the strings of the offspring are occasionally altered. Its main purpose is to maintain diversity in the population. Here, the polynomial mutation was performed (Goldberg, 1989).

\subsection{Selection of pareto optimal solutions}

The optimization algorithm generated a lot of optimized hydrofoils with varied GR and cavitation characteristics. Each of the generated hydrofoils is better off with regard to one objective but is worse off with regard to another objective. Hence, they are called Pareto-optimal solutions. The hydrofoil with the highest GR over a wide range of AOA was selected to model the optimized turbine.

\subsection{The stopping criterion}

In GAs, either of the two following approaches is employed to set the stopping criterion. First, the algorithm is set to stop if the solutions of the next generation are insignificant compared to that of its previous generation. Second, the maximum number of generations is set to a predefined value. Here, the second approach was adopted and the stopping criterion was set to 30 generations. It is a straightforward approach and was employed by taking into account the computational time. The optimized hydrofoils were generated until the stopping criterion was met. For more details, refer to (Deb, 2009)

\section{Hydrofoil Shape Optimization Strategies}

The 2-D shape optimization of hydrofoil was done by considering three parameters, namely, $C_{L}, C_{D}$ and minimum pressure coefficient $\left(C_{P \min }\right)$. Here, optimization was carried out to maximize $C_{L}$ and minimize $C_{D}$ in order to increase the output power of a tidal stream turbine. The optimization algorithm also sought to increase the $C_{P \min }$ to improve its cavitation performance 


\begin{tabular}{|c|c|c|c|c|}
\hline Generation & Mean $C_{L}$ & Mean $C_{D}$ & Mean $C_{P \min }$ & Number of optimized hydrofoils \\
\hline 1st & 1.1337 & 0.014 & -2.3882 & 11 \\
\hline 5th & 1.1664 & 0.012 & -2.115 & 18 \\
\hline 10th & 1.1866 & 0.0123 & -2.0462 & 24 \\
\hline 15th & 1.1789 & 0.0122 & -2.0458 & 22 \\
\hline 20th & 1.1721 & 0.012 & -2.0645 & 18 \\
\hline 25th & 1.1656 & 0.012 & -2.0568 & 20 \\
\hline 30th & 1.1713 & 0.0119 & -2.0899 & 20 \\
\hline
\end{tabular}

Table 1: Comparison of optimization results at different generations

(Luo et al., 2014). Considering different AOA in the optimization algorithm generates optimized hydrofoils with different performance characteristics. Mukesh et al. (2014) carried out the shape optimization for an AOA of $5^{\circ}$. But (Luo et al., 2014; Prosser et al., 2010) argued that the shape optimization done at a single AOA often results in poor performance at off-design conditions. Hence (Luo et al., 2014) performed the optimization for AOA of $0^{\circ}, 6^{\circ}$ and $12^{\circ}$ while (Prosser et al., 2010) performed for AOA $1.5^{\circ}, 4^{\circ}$ and $7^{\circ}$. The AOA in the optimization algorithm must therefore be chosen with care and hence, its effect was studied in the following sections. The effect of number of objectives and its implication on the solutions was also studied.

\subsection{Three dimensional (3-D) optimization}

The main idea behind 3-D optimization was to handle each of the objectives individually, i.e. $C_{L}, C_{D}$ and $C_{P \min }$. Two cases were considered to study the effect of AOA. In the first case a wide range of five AOA were considered and in the second case three AOA were considered. In the first case, the objective is to maximize the average $C_{L}$ and $C_{P \min }$ and to minimize the average $C_{D}$ at $\mathrm{AOA}$ of $0^{\circ}, 3^{\circ}, 6^{\circ}, 9^{\circ}$ and $12^{\circ}$ for the objective functions shown in equations [3 - 5]. $C_{L}, C_{D}$ and $C_{p_{\text {min }}}$ were averaged to give equal weightage to all the AOA considered.

$$
\begin{gathered}
\frac{\Sigma C_{L_{, A O A}}}{N} \\
\frac{\Sigma C_{D_{, A O A}}}{N} \\
\frac{\Sigma C_{P \min , A O A}}{N}
\end{gathered}
$$

where $\mathrm{N}$ is the number of different AOA considered. Table 1 shows the comparison of performance characteristics of the hydrofoils that were generated in the first case. Every generation consist of 80 hydrofoils. The mean values were taken in order to assess the efficacy of the 

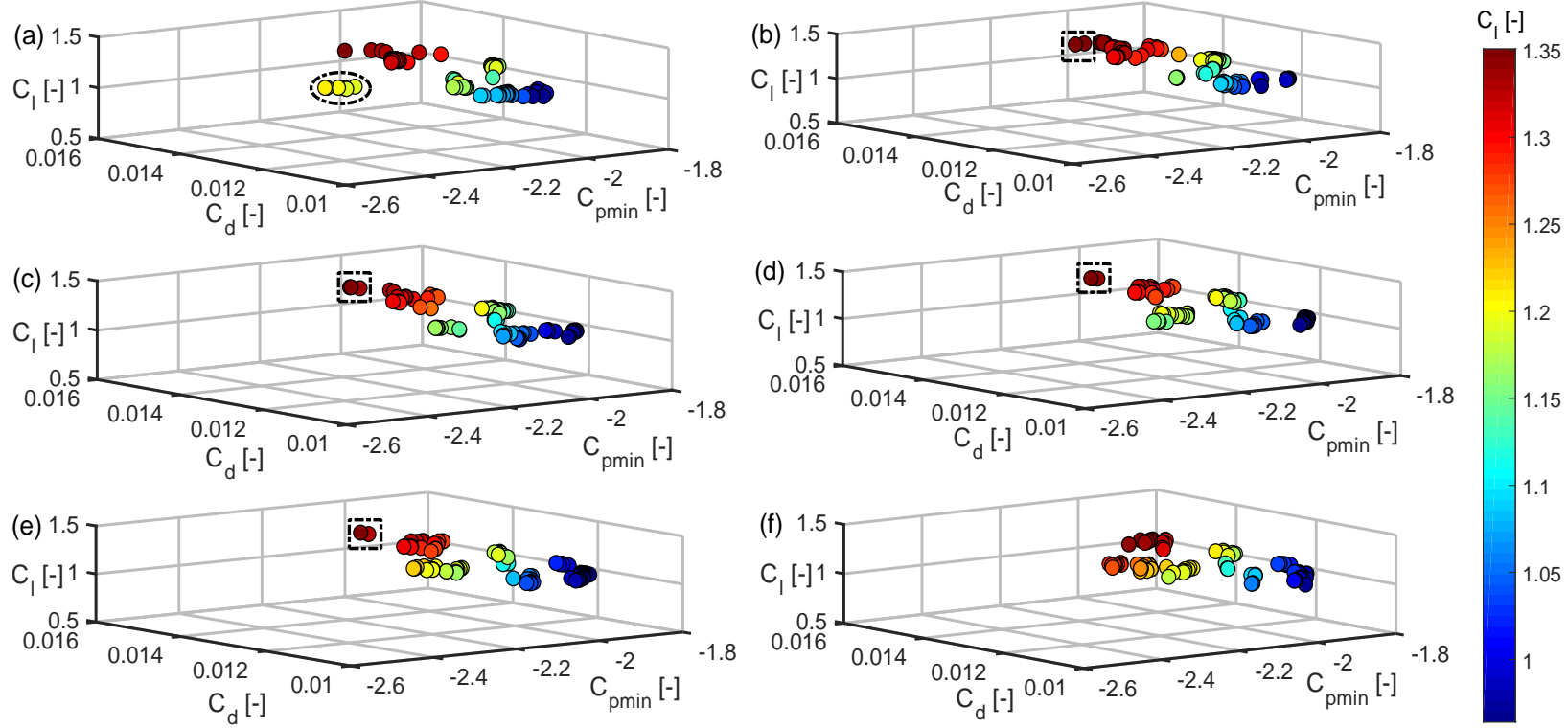

Figure 6: 3D Pareto optimal graph of the different generations, where (a) represents the 5th, (b) represents the 10th, (c) represents the 15th, (d) represents the 20th, (e) represents the 25th and (f) represents the 30th generations

optimization algorithm in generating hydrofoils with higher $C_{L}$ and $C_{P \min }$ and lower $C_{D}$. The hydrofoils with $C_{L}$ and $C_{P \min }$ greater than the reference hydrofoil and $C_{D}$ lesser than the reference hydrofoil were designated as optimized hydrofoils in each generation. The mean $C_{L}$ and $C_{P \min }$ of the hydrofoils in the last generation is higher than that of the first generation and the mean $C_{D}$ is lower which indicates that the optimization algorithm could generate better hydrofoils as the execution of the optimization code progresses. The 3D Pareto-optimal graphs of the first case for different generations are shown in Figure 6. The hydrofoil characteristics are colour-coded according to their $C_{L}$ values. Figure 6 (a) shows performance characteristics of optimized hydrofoils obtained in the 5 th generation. Seven hydrofoils are shown within a dotted black ellipse, which have poor cavitation characteristics relative to the others in the same generation. Their $C_{P \min }$ ranges from -2.438 to $-2.359, C_{D}$ ranges from 0.117 to 0.012 , and $C_{L}$ ranges from 1.19 to 1.22 . However, since some hydrofoils overlap another, only four of them are visible. In the 10th generation as shown in Figure 6 (b) the seven hydrofoils were excluded and were replaced with hydrofoils having better cavitation characteristics. Three hydrofoils that are shown within dotted black rectangles in Figure 6 (b-e) having $C_{L}$ ranging from 1.35 to 1.4 and $C_{D}$ ranging from 0.014 to 0.0142 were retained until the 25 th generation. Although they have the worst $C_{D}$ values, they were retained until the 25th generation owing to their high $C_{L}$ values. They were eventually replaced with hydrofoils having lower $C_{D}$ and higher $C_{P \min }$ in the 30th generation as shown in Figure 6 (f). In course of the execution of the optimization code, the $C_{D}$ of the generated hydrofoils gradually became lesser compared to its preceding 

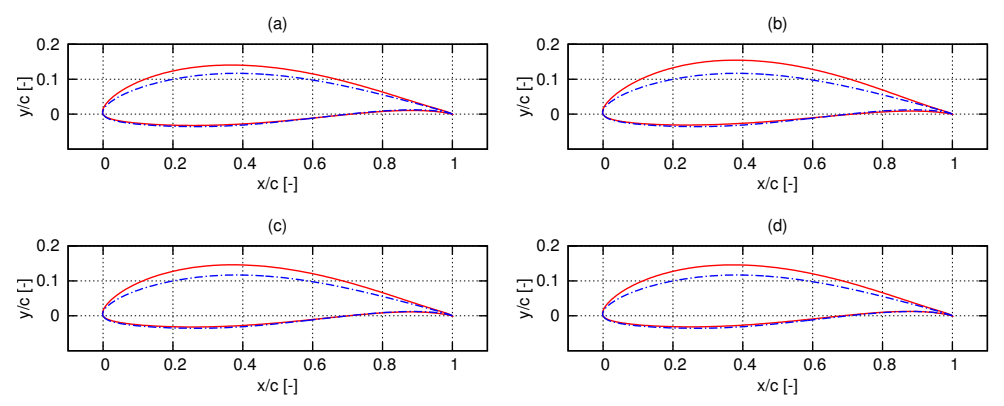

Figure 7: Samples of the optimized hydrofoils of the first case, where the red solid lines denote the optimized hydrofoil and the blue dash-dot lines denote the original hydrofoil

generations. Four hydrofoils with the highest GR are shown in Figure 7. Figure $7(a)$ shows an optimized hydrofoil ( HF-3D-1-1), which has maximum thickness $\left(t_{\max }\right)$ of $17.09 \%$ at $33.7 \%$ of the chord length from the leading edge $(0.337 c)$. Here HF refers to hydrofoil, 3D refers to three dimensional optimization, the first 1 refers to the first case and the second 1 refers to the first optimized hydrofoil. It can be seen in Figure $8(a)$ that the HF-3D-1-1 has a higher GR from $\mathrm{AOA}=0^{\circ}$ to almost $2^{\circ}$ and from $\mathrm{AOA}=6.1^{\circ}$ to $12^{\circ}$. Figure $9(a)$ shows that $C_{P \min }$ of the optimized hydrofoil has been improved at higher AOA ranging from $5^{\circ}$ to $12^{\circ}$. Figure $7(b)$ shows the second optimized hydrofoil (HF-3D-1-2), which has $t_{\max }$ of $18.26 \%$ at $0.347 c$. It can be seen from Figure $8(b)$ that the optimized hydrofoil has a higher GR from AOA $=0^{\circ}$ to $1^{\circ}$ and from $\mathrm{AOA}=6.2^{\circ}$ to $12^{\circ}$. Figure $9(\mathrm{c})$ shows that the cavitation performance of the optimized hydrofoil is closer to that of the reference hydrofoil at lower AOA and from AOA of $5^{\circ}$ the cavitation performance has been greatly improved. Figure $7(c)$ shows the third optimized hydrofoil (HF-3D-1-3), which has $t_{\max }$ of $17.58 \%$ at $0.339 c$. It can be seen from Figure $8(c)$ that the optimized hydrofoil has a higher GR from $\mathrm{AOA}=0^{\circ}$ to $1^{\circ}$ and from $\mathrm{AOA}=6.2^{\circ}$ to $12^{\circ}$. Figure $9(b)$ shows that the cavitation performance of the optimized hydrofoil is closer to the reference hydrofoil at lower $\mathrm{AOA}$ and from $\mathrm{AOA}=5^{\circ}$ the cavitation performance has been greatly improved. Figure $7(d)$ shows the fourth optimized hydrofoil(HF-3D-1-4) has $t_{\text {max }}$ of $17.5 \%$ at $0.337 c$. Although Figures $8(d)-9(d)$ show that the GR and $C_{p_{\text {min }}}$ were not improved at lower AOA, it performs well at higher AOA. In the first case, hydrofoils were produced that performed consistently well at wide range of AOA. The GR and the cavitation performance were improved at higher AOA. However, the maximum GR of the optimized hydrofoils were lower than the reference hydrofoil, which is undesirable.

Since the maximum GR of the reference and the optimized hydrofoils in the previous case lie between AOA of $4^{\circ}$ and $6^{\circ}$, the optimization was done at these AOA with a focus on increasing 

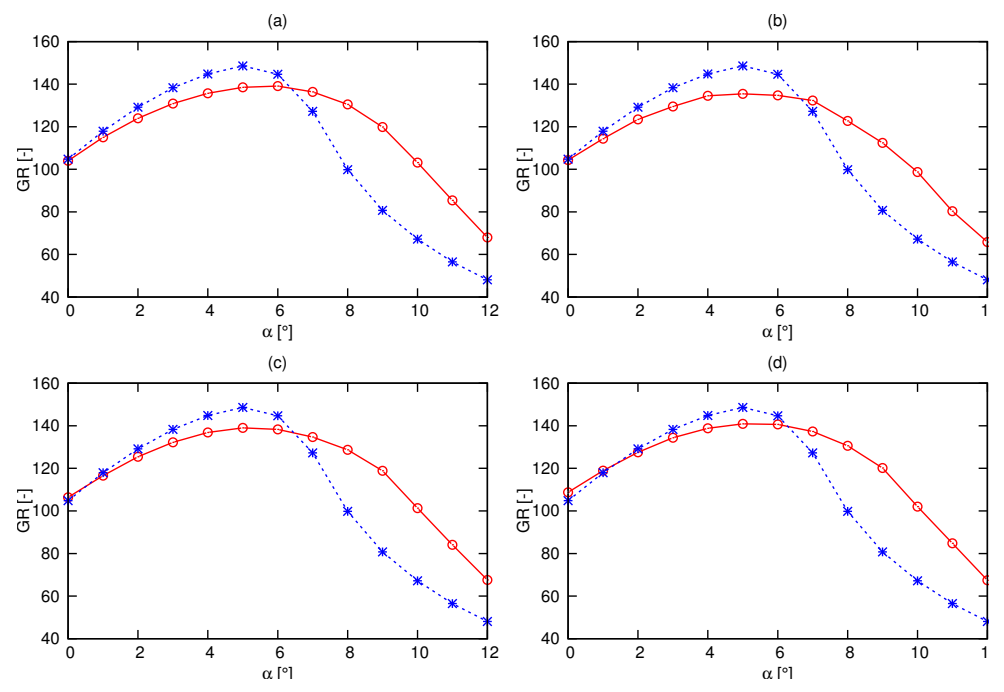

Figure 8: Comparison of the GR of the reference and optimized hydrofoils of the first case of 3D optimization, where the red and blue lines denote optimized and reference hydrofoil respectively
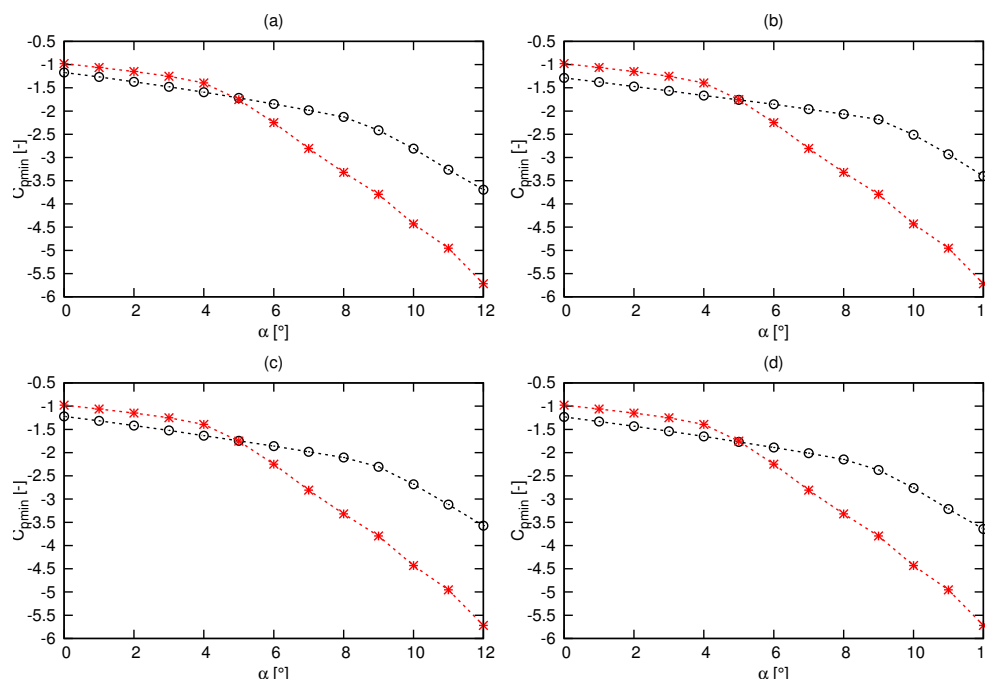

Figure 9: Comparison of the $c_{p m i n}$ of the reference and optimized hydrofoils of the first case of $3 \mathrm{D}$ optimization, where the red and blue lines denote optimized and reference hydrofoil respectively 

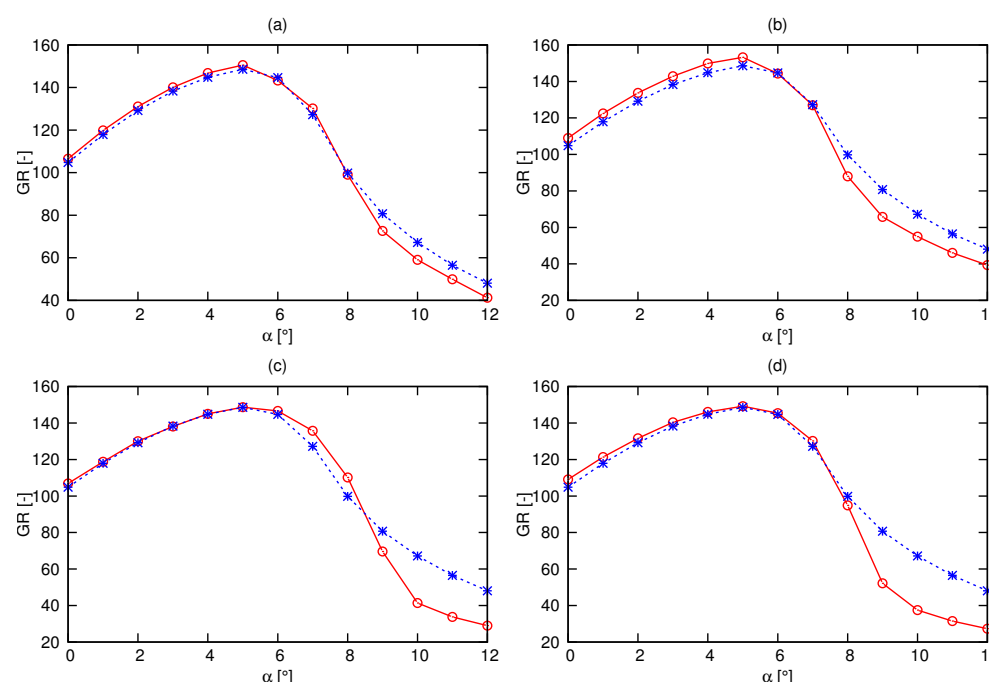

Figure 10: Comparison of the GR of the reference and optimized hydrofoils of the second case of 3D optimization, where the red and blue lines denote optimized and reference hydrofoil respectively

the maximum GR. Equations 3, 4 and 6 are calculated for AOA of $4^{\circ}, 5^{\circ}$ and $6^{\circ}$. The optimized hydrofoils of this case have higher GR at smaller AOA of the hydrofoils obtained in the first case wherein the optimized hydrofoils had better performance characteristics only at higher AOA. Further, the Pareto optimal solutions are not as diverse as the first case.

\subsection{Two dimensional (2-D) optimization}

It is possible that the process of evolution of the optimized hydrofoils was slowed down on account of it being a high-dimensional multi-objective optimization problem. The number of objectives was, therefore, reduced to two by considering $\mathrm{C}_{p_{\min }}$ and GR as the optimization objectives as shown equations 5 and 6 respectively.

$$
\frac{\Sigma G R_{, A O A}}{N}
$$

where $\Sigma G R_{, A O A}$ is the summation of GR at different AOA. In this case the summation of GR and $C_{P \min }$ are calculated at AOA of $0^{\circ}, 3^{\circ}, 6^{\circ}, 9^{\circ}$ and $12^{\circ}$. Table 2 shows the comparison of the performance characteristics of the hydrofoils in different generations. The mean GR and $C_{P \min }$ values show only the general behaviour of the optimization algorithm, which sporadically increase and decrease for two reasons. Firstly, optimization was done by considering two conflicting objectives i.e. improvement in one objective is achieved at the expense of the other objective. Secondly, the crowding distance operator was used to diversify the solutions at the end of each generation. In Table 2 it can be seen that in the 5 th generation the number of optimized hydrofoils is higher than all the other generations. For better clarity refer to Figure 12 (a) 

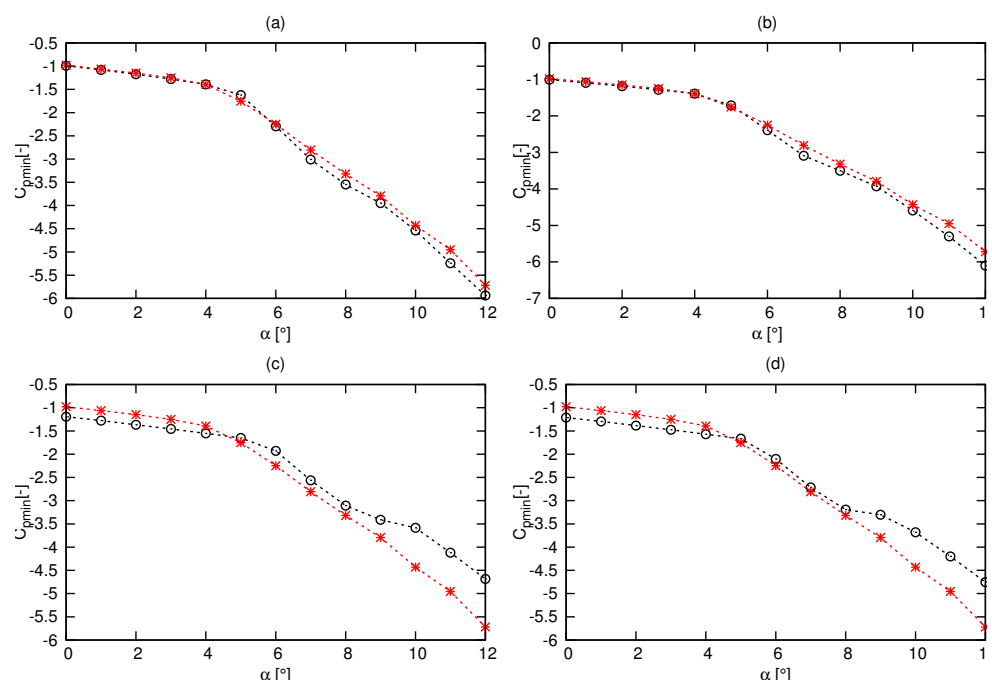

Figure 11: Comparison of the $C_{P \min }$ of the reference and optimized hydrofoils of the second case of 3D optimization, where the red and blue lines denote optimized and reference hydrofoil respectively

which shows that the hydrofoil characteristics of this generation are bunched together meaning that some of the optimized hydrofoils are almost similar. The duplication of hydrofoils is avoided by using the crowding distance operator whose primary task is to diversify the Pareto optimal solutions as shown in Figure 12. The distribution of the performance characteristics of hydrofoils in the 30th generation (Figure 12 (f)) is the most diverse compared to the other generations (Figure 12 (a)-(e)). With diverse solutions, the designer will have a lot of hydrofoils to choose from based on their specific requirements.

Four hydrofoils with the highest GR in the 30th generation are shown in Figure 13. The GR of hydrofoil (HF-2D-1-1) whose $t_{\max }$ is $15.09 \%$ at $0.303 c$ is shown Figure $13(a)$. The GR of this hydrofoil is higher than that of the reference hydrofoil from $\mathrm{AOA}=0^{\circ}$ to $2^{\circ}$ and

\begin{tabular}{|c|c|c|c|}
\hline Generation & Mean Glide Ratio & Mean $C_{p_{\min }}$ & Number of optimized hydrofoils \\
\hline 1st & 94.4015 & -2.1861 & 21 \\
\hline 5th & 98.1213 & -1.9701 & 34 \\
\hline 10th & 96.5394 & -1.9297 & 27 \\
\hline 15th & 97.9764 & -1.9464 & 33 \\
\hline 20th & 97.6847 & -1.9418 & 33 \\
\hline 25th & 96.7179 & -1.937 & 30 \\
\hline 30th & 96.5764 & -1.9312 & 30 \\
\hline
\end{tabular}

Table 2: Comparison of optimization results at different generations in the first case of 2D optimization 

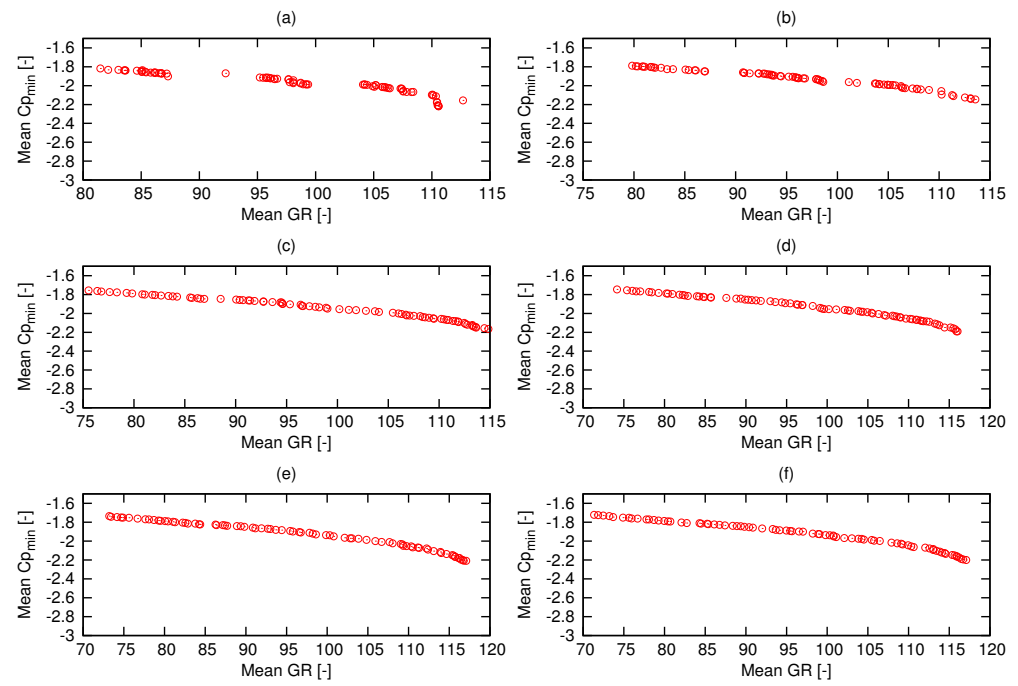

Figure 12: Pareto-optimal graphs for the different generations in the first case of 2D optimization, where (a) represents the 5th, (b) represents the 10th, (c) represents the 15th, (d) represents the 20th, (e) represents the 25th and (f) represents the 30th generations

from $\mathrm{AOA}=6.2^{\circ}$ to $12^{\circ}$ as shown in Figure $14(a)$. The cavitation performance is better from AOA greater than $5^{\circ}$ as shown in Figure $15(a)$. The second optimized hydrofoil (HF-2D-12 ) in Figure $13(b)$ has $t_{\max }$ of $17.09 \%$ at $0.337 c$. The GR of this hydrofoil is higher than the reference hydrofoil from $\mathrm{AOA}=0^{\circ}$ to $2^{\circ}$ and from $\mathrm{AOA}=6.2^{\circ}$ to $12^{\circ}$ as shown in Figures $15(b)$. The cavitation performance is better from AOA greater than $5^{\circ}$ as shown in Figure $14(b)$. The third optimized hydrofoil (HF-2D-1-3) shown in Figure $13(c)$ has $t_{\max }$ of $17.5 \%$ at $0.339 c$ as shown in Figure $15(c)$. The GR of the hydrofoil is higher than the reference hydrofoil from $\mathrm{AOA}=0^{\circ}$ to $2^{\circ}$ and from $\mathrm{AOA}=6.2^{\circ}$ to $12^{\circ}$ and the cavitation performance is better from AOA greater than $5^{\circ}$ as shown in Figure $15(c)$. The fourth optimized hydrofoil (HF-2D-1-4) shown in Figure $13(d)$ has $t_{\max }$ of $17.5 \%$ at $0.337 c$ as shown in Figure $15(d)$. The maximum GRs of the hydrofoils obtained in this case are lower than the reference hydrofoil, but they have better cavitation characteristics at higher AOA as shown in Figure $14(d)$. By reducing the number of objectives to two, a marked improvement in the performances of the hydrofoils could be noticed.

Further, the maximum GRs lie between AOA 4 and 6. Hence, 2D optimization was done for these AOA. Equations 5 and 6 are calculated for AOA of $4^{\circ}, 5^{\circ}$ and $6^{\circ}$. Four hydrofoils with the highest GR are shown in Figure 16. The GR of HF-2D-2-1 that is shown in Figure $16(a)$ is higher than that of the reference hydrofoil from AOA $0^{\circ}$ to $8^{\circ}$ and the $C_{P \text { min }}$ is greater from AOA greater than $5^{\circ}$ as shown in Figure $18(a)$ and Figure $17(a)$ respectively. The GR of the second hydrofoil (HF-2D-2-2) is higher than that of the reference hydrofoil from AOA $0^{\circ}$ to $9.8^{\circ}$ and the cavitation performance is better from AOA greater than $5^{\circ}$ as shown in Figure $18(b)$ and Figure $17(b)$ respectively. The third optimized hydrofoil (HF-2D-2-3) whose $t_{\max }$ is $17.13 \%$ 

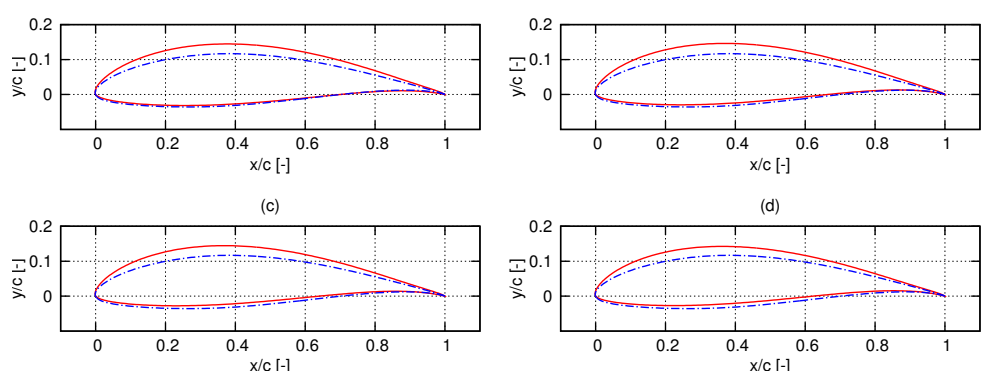

Figure 13: Samples of optimized hydrofoils of the first case of 2D optimization
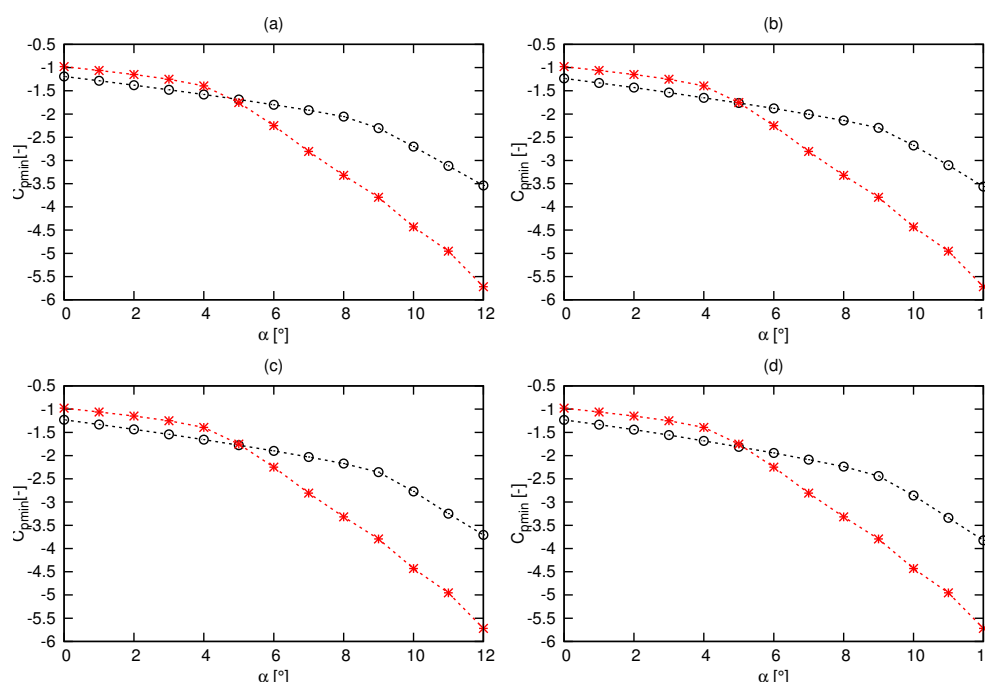

Figure 14: Comparison of the $C_{P \min }$ of reference and optimized hydrofoils of the first case of 2D optimization 

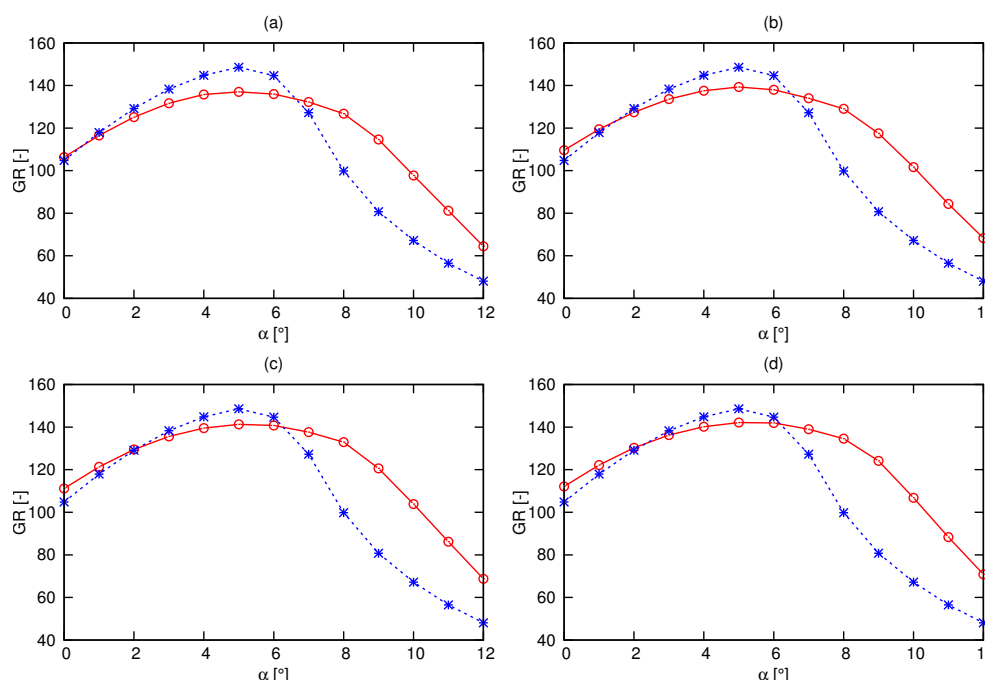

Figure 15: Comparison of the GR of reference and optimized hydrofoils of the first case of 2D optimization
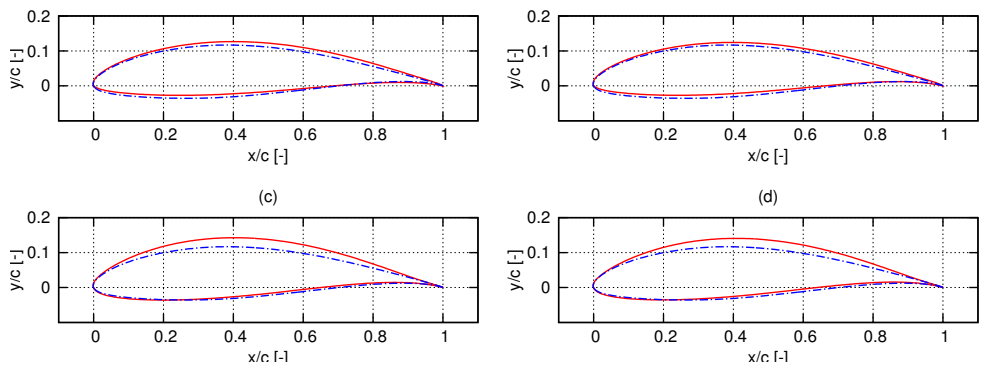

Figure 16: Samples of optimized hydrofoils of the second case of 2D optimization

at $0.347 c$ is shown in Figure $13(c)$. The GR of this hydrofoil is significantly higher than the reference hydrofoil from $\mathrm{AOA} 0^{\circ}$ to almost $10^{\circ}$ and the cavitation performance was better than the reference hydrofoil from AOA greater than $5^{\circ}$. The performance characteristics of the fourth hydrofoil (HF-2D-2-4) are not as good as the previous two hydrofoils but still are better than the reference hydrofoil, especially with regard to the GR from AOA $0^{\circ}$ to $8^{\circ}$. The GRs of the hydrofoils obtained in this case are also higher than that of all the optimized hydrofoils in the previous three cases. On the other hand, the cavitation performances are not as good as that of the optimized hydrofoils obtained in the first cases of 2D and 3D optimization. Some of the optimized hydrofoils obtained in this case have GRs greater than the reference hydrofoil from AOA raning from $0^{\circ}$ to $10^{\circ}$. Further, the GRs of the hydrofoils are higher than those obtained in the other optimization cases. On the other hand, the cavitation characteristics were almost the same from $0^{\circ}$ to $5^{\circ}$ and after that they were improved until AOA of $12^{\circ}$. Although hydrofoils were generated with higher $C_{L}$, lower $C_{D}$ and higher $C_{P \min }$, the evolution of optimized hydrofoils was slow. Further, the influence of the crowding distance operator was apparently 

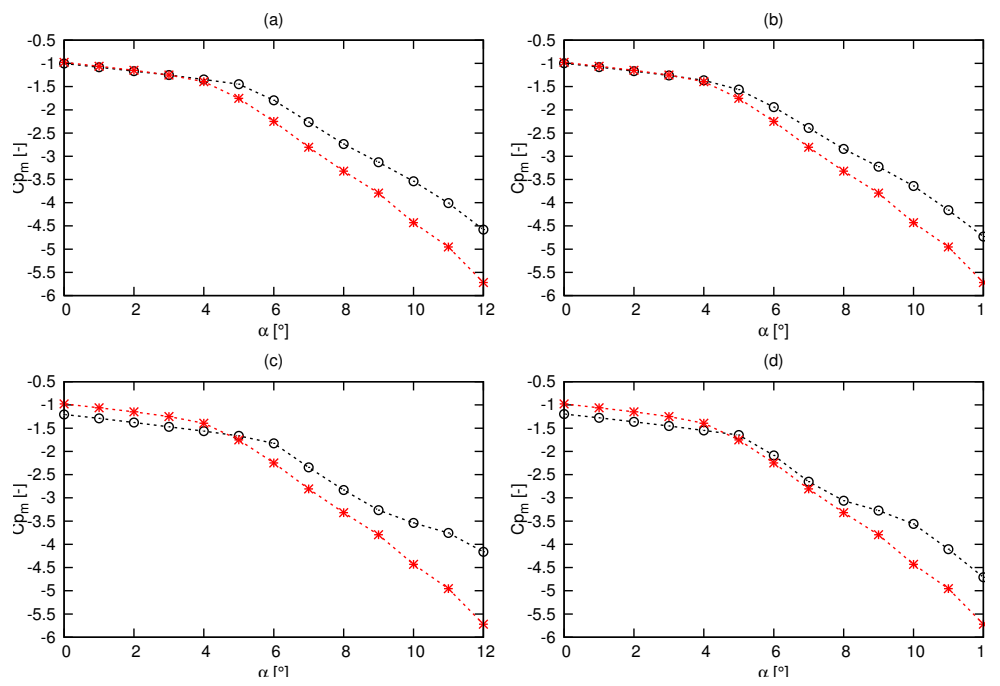

Figure 17: Comparison of the GR of the reference and optimized hydrofoils of the second case of $2 \mathrm{D}$ optimization, where the red dotted-lines with astricks represent the reference hydrofoil and the blue dashed-line with open circles represent optimized hydrofoil
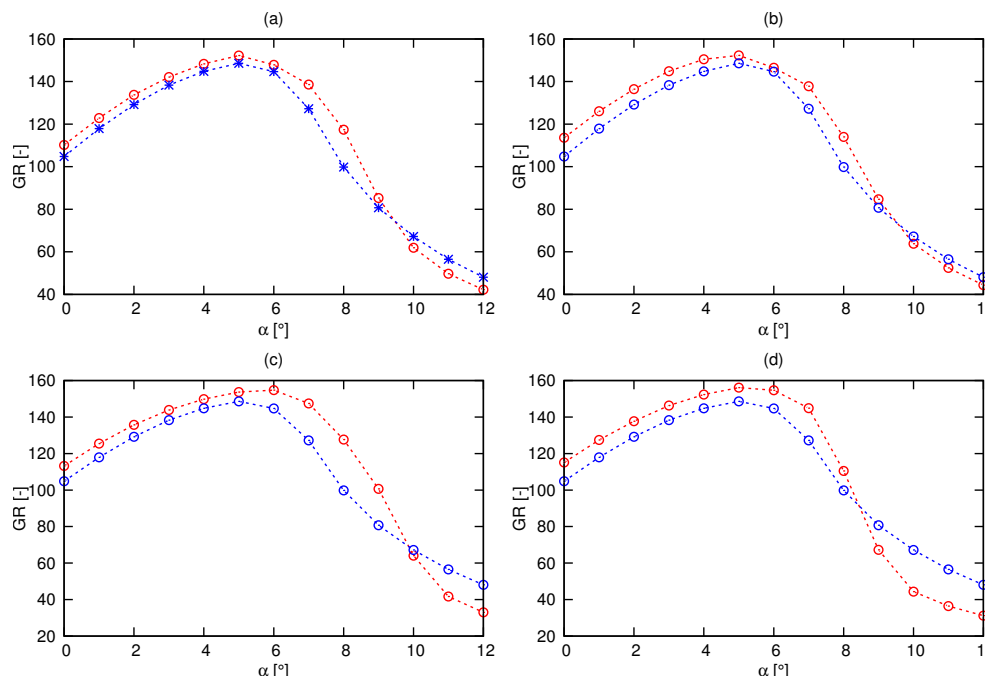

Figure 18: Comparison of the $C_{P \min }$ of the reference and optimized hydrofoils of the second case of $2 \mathrm{D}$ optimization 
insignificant in the 3D optimization.

\section{4 conclusion}

The onus is on the designer of the tidal stream turbines to select hydrofoils based on their specific requirements.

An in-house MATLAB code was developed based on (Deb et al., 2002b) to perform shape optimization of hydrofoils by employing NSGA-II algorithm and coupled with a flow solver. The hydrofoil was parameterized using $6^{\text {th }}$ order Bezier curves. In that case NACA68.. was taked as a reference hydrofoil. The maximum normalized deviation of the Bezier parameterized reference hydrofoil from the original hydrofoil was accurate enough to carry out shape optimization.

For the chossen solver, The 2D panel method XFOIL solver and CFD results of Lift and drag coefficients of the reference hydrofoil were compared with the experimental measurments. The results for CFD is more acurate but also more time consuming, thus the XFOIL solver was chosen on account of its simplicity and its ability to give acceptable results within a short span of time compare to CFD.

A comparative study on the optimization was done by considering two methods. 3D optimization, three objective i.e. $C_{L}, C_{D}$ and $C_{p_{m i n}}$ were the optimization objectives and 2D optimization, the number of objectives was reduced to two by unifying $C_{L}$ and $C_{D}$ as GR. The GR of the hydrofoils was further improved by performing optimization at AOA of $4^{\circ}, 5^{\circ}$ and $6^{\circ}$ than on performing at $\mathrm{AOA}$ of $0^{\circ}, 3^{\circ}, 6^{\circ}, 9^{\circ}$ and $12^{\circ}$.

This was because the maximum glide ratio of refrence hydrofoil fell within the range of $4^{\circ}$ and $6^{\circ}$. However, the cavitation characteristics were better at higher AOA in the first case.

The multi-objective optimization performed in the present work effectively improved the GR of the hydrofoils from AOA of $0^{\circ}$ to almost $10^{\circ}$. The cavitation performance was mainly improved from AOA $5^{\circ}$ to $12^{\circ}$. The solutions of optimization are not unique. Different hydrofoils with different performance characteristics were obtained, but a lot of them performed better than NACA 63815.

\section{References}

Abbott, I. H. and Von Doenhoff, A. E. (1959). Theory of wing sections, including a summary of airfoil data. Courier Corporation.

Baskar, S., Tamilselvi, S., and Varshini, P. (2015). Matlab code for constrained nsga ii. https://de.mathworks.com/matlabcentral/fileexchange/ 49806 -matlab-code-for-constrained-nsga-ii-dr-s-baskar--s-tamilselvi-and[Online; accessed October 2017]. 
Batten, W., Bahaj, A., Molland, A., and Chaplin (2007). Experimentally validated numerical method for the hydrodynamic design of horizontal axis tidal turbines. Ocean engineering, 34(7):1013-1020.

Batten, W., Bahaj, A., Molland, A., and Chaplin, J. (2006). Hydrodynamics of marine current turbines. Renewable energy, 31(2):249-256.

Ching-Yeh, H., Jia-Lin, W., and Chang, S.-F. (2006). Design and optimization method for a two-dimensional hydrofoil. Journal of Hydrodynamics, Ser. B, 18(3):323-329.

De Boor, C., De Boor, C., Mathématicien, E.-U., De Boor, C., and De Boor, C. (1978). A practical guide to splines, volume 27. Springer-Verlag New York.

Deb, K. (2009). Multi-Objective Optimization Using Evolutionary Algorithms. Wiley.

Deb, K., Pratap, A., Agarwal, S., and Meyarivan, T. (2002a). A fast and elitist multiobjective genetic algorithm: Nsga-ii. IEEE transactions on evolutionary computation, 6(2):182-197.

Deb, K., Pratap, A., Agarwal, S., and Meyarivan, T. (2002b). A fast and elitist multiobjective genetic algorithm: Nsga-ii. IEEE Transactions on Evolutionary Computation, 6(2):182-197.

Drela, M. (1989). Xfoil: An analysis and design system for low reynolds number airfoils. In Low Reynolds number aerodynamics, pages 1-12. Springer.

Goldberg, D. (1989). Genetic Algorithms in Search, Optimization, and Machine Learning. Artificial Intelligence. Addison-Wesley Publishing Company.

Goundar, J. N. and Ahmed, M. R. (2013). Design of a horizontal axis tidal current turbine. Applied energy, 111:161-174.

Goundar, J. N., Ahmed, M. R., and Lee, Y.-H. (2012). Numerical and experimental studies on hydrofoils for marine current turbines. Renewable energy, 42:173-179.

Kennedy, J. (2011). Particle swarm optimization. In Encyclopedia of machine learning, pages 760-766. Springer.

Kennedy, J. and Eberhart, R. (1995). Particle swarm optimization. In Proceedings of ICNN'95 - International Conference on Neural Networks, volume 4, pages 1942-1948 vol.4.

Konak, A., Coit, D. W., and Smith, A. E. (2006). Multi-objective optimization using genetic algorithms: A tutorial. Reliability Engineering \& System Safety, 91(9):992-1007.

Litvinov, W. (1995). On the optimal shape of a hydrofoil. Journal of optimization theory and applications, 85(2):325-345. 
Liu, P. and Veitch, B. (2012). Design and optimization for strength and integrity of tidal turbine rotor blades. Energy, 46(1):393-404.

Luo, X.-q., Zhu, G.-j., and Feng, J.-j. (2014). Multi-point design optimization of hydrofoil for marine current turbine. Journal of Hydrodynamics, Ser. B, 26(5):807-817.

Menter, F. (1993). Zonal two equation kw turbulence models for aerodynamic flows. In 23rd fluid dynamics, plasmadynamics, and lasers conference, page 2906.

Molland, A., Bahaj, A., Chaplin, J., and Batten, W. (2004). Measurements and predictions of forces, pressures and cavitation on 2-d sections suitable for marine current turbines. Proceedings of the Institution of Mechanical Engineers, Part M: Journal of Engineering for the Maritime Environment, 218(2):127-138.

Mukesh, R., Lingadurai, K., and Selvakumar, U. (2014). Airfoil shape optimization using non-traditional optimization technique and its validation. Journal of King Saud UniversityEngineering Sciences, 26(2):191-197.

Nemec, M., Zingg, D. W., and Pulliam, T. H. (2004). Multipoint and multi-objective aerodynamic shape optimization. AIAA journal, 42(6):1057-1065.

Ouyang, H., Weber, L., and Odgaard, A. (2006). Design optimization of a two-dimensional hydrofoil by applying a genetic algorithm. Engineering Optimization, 38(5):529-540.

Paolo, Olivucci, S., and Gaggero (510 June 2016). A framework for the design by optimization of hydrofoils under cavitating conditions. In VII European Congress on Computational Methods in Applied Sciences and Engineering, ECCOMAS Congress.

Patankar, S. V. (2016). Computational Methods for Fluid Dynamics. 9th Indian Reprint, Taylor\& Francis.

Peigin, S. and Epstein, B. (2004). Robust optimization of 2d airfoils driven by full navier-stokes computations. Computers \& fluids, 33(9):1175-1200.

Prosser, D., Guerrette, J., Regula, A., and Ozgur, M. (2010). Airfoil shape optimization using xfoil and modified naca parameters.

Ribeiro, A. F., Awruch, A. M., and Gomes, H. M. (2012). An airfoil optimization technique for wind turbines. Applied Mathematical Modelling, 36(10):4898-4907.

Shahrokhi, A. and Jahangirian, A. (2007). Airfoil shape parameterization for optimum navierstokes design with genetic algorithm. Aerospace science and technology, 11(6):443-450.

Tahani, M. and Babayan, N. (2017). Optimum section selection procedure for horizontal axis tidal stream turbines. Neural Computing and Applications. 
Zhang, D.-S., Chen, J., Shi, W.-D., Shi, L., and Geng, L.-L. (2016). Optimization of hydrofoil for tidal current turbine based on particle swarm optimization and computational fluid dynamic method. Thermal Science, 20(3):907-912. 